\title{
Giant Epidermoid Cyst of the Thigh
}

\section{NH Mohamed Haflah, MS Orth, AY Mohd Kassim, MS Orth, M Hassan Shukur, MS Orth}

Department of Orthopaedics and Traumatology, Faculty of Medicine, Universiti Kebangsaan Malaysia

\begin{abstract}
Epidermoid cyst is a common benign cutaneous swelling frequently encountered in surgical practice. It usually presents as a painless lump frequently occurring in hairbearing areas of the body particularly the scalp, scrotum, neck, shoulder and back. Giant epidermoid cysts commonly occur in hairy areas such as the scalp. We present here the case of a rare occurrence of a giant epidermoid cyst in the less hairy area of the right upper thigh mimicking a soft tissue sarcoma. Steps are highlighted for the management of this unusual cyst.
\end{abstract}

Key Words:

Cystic Lesion, Epidermoid Cyst, Giant, Thigh

\section{INTRODUCTION}

Epidermoid cyst is a common cutaneous lesion, typically presenting as a small painless lump in hair-bearing areas of the body. Giant epidermoid cysts are most commonly reported in the scalp ${ }^{1}$ and infrequently in less hairy areas of the gluteal ${ }^{2,3}$ and forearm ${ }^{4}$. We report a rare case of giant epidermoid cyst in the thigh, also less hairy part of the body. With such a large size, the swelling appeared to be a soft tissue sarcoma and the diagnosis of epidermoid cyst was not considered initially.

\section{CASE REPORT}

A 65-year-old female complained of swelling on the outer aspect of her right thigh. The swelling existed as a small painless lump for the previous 10 years; recently, it grew bigger thereby causing much discomfort with a cramping sensation in her right thigh over the previous six months. Consequently, she had difficulty, not only in sitting, but also carrying out daily activities of daily living. She reported no past history of injections to the thigh. There was also no history of loss of weight or appetite.

On examination, there was a firm non-tender lump measuring $10 \times 11 \mathrm{~cm}$ over the lateral aspect of the right thigh. The overlying skin was normal and the most proximal edge of the cyst was approximately $5 \mathrm{~cm}$ from the greater trochanter. The lump was not tense and there was no punctum or scar. The lump was not adhered to the skin or attached to the underlying muscle; both fluctuation and transillumination tests were negative and there was no inguinal lymphadenopathy. Distal pulses of the right leg were normal and there was no neurological deficit. At this point, our main concern was to exclude malignant soft tissue sarcoma due to the large size of the growth (more than $8 \mathrm{~cm}$ in dimension), as recommended by Hadju's clinical prediction rule for soft tissue sarcoma.

Magnetic resonance imaging (MRI) revealed a well-defined lobulated encapsulated mass within the subcutaneous adipose tissue over the posterolateral aspect of the right thigh. The mass measured $8.5 \mathrm{~cm} \times 6.0 \mathrm{~cm} \times 7.5 \mathrm{~cm}$. On T1weighted images, the mass was homogenously hypointense, whilst on T2- weighted images it was homogenously hyperintense. Signal was not suppressed in the fat saturation images (Figure 1), and there was neither solid component nor calcification within the mass. These changes were consistent with a benign cystic mass.

As soft tissue sarcoma remained to be excluded, a wide local excision of the mass was planned. Intraoperatively, the excised mass was an encapsulated cyst (Figure 2) containing soft white cheesy material consistent with an epidermoid cyst. Histopathology examination showed a thin fibrous cyst wall lined by stratified squamous epithelium and focally by foamy macrophages confirming a diagnosis of epidermoid cyst (Figure 3).

Post-operative recovery was uneventful. At one year followup, the patient showed no evidence of recurrence.

\section{DISCUSSION}

The term epidermoid cyst is sometimes used interchangeably with sebaceous cyst. However, epidermoid cyst does not originate from sebaceous gland ${ }^{1}$. Epidermoid cysts are lined with stratified squamous epithelium. Keratin debris and epithelium accumulate in the cyst, giving rise to the cheesy appearance with a distinct odour.

Epidermoid cysts typically present as painless small lumps in hair-bearing areas of the body particularly the scalp, neck, shoulder and back. It may increase in size over time 


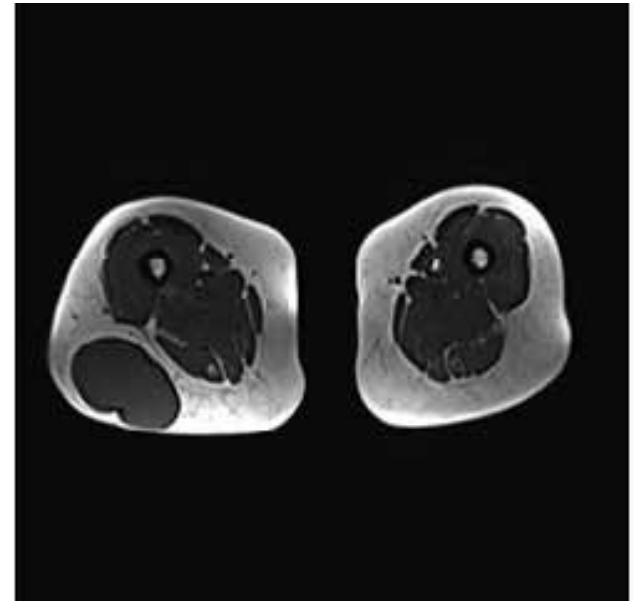

Fig. 1: MRI of the upper femur. Axial view of T1-weighted image revealed homogenously hypointense mass.

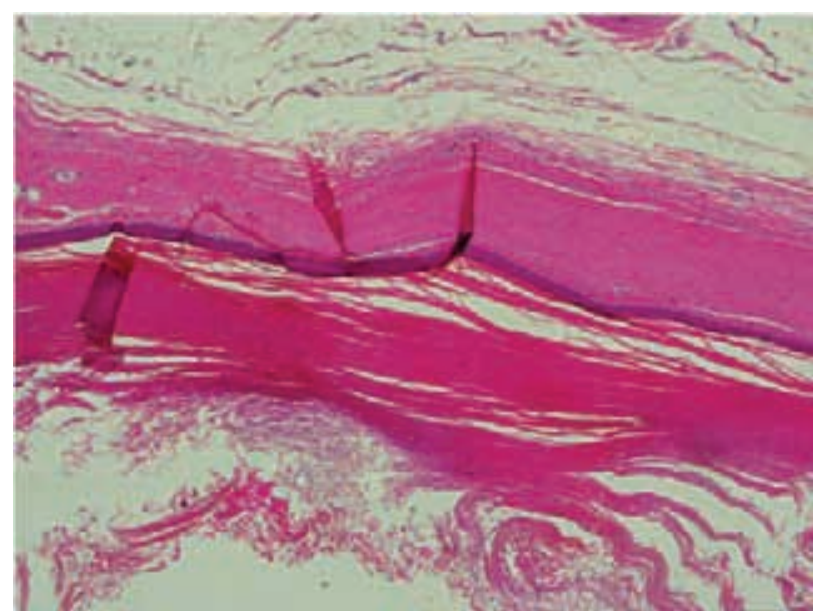

Fig. 3: Histological examination showed thin fibrous cyst lined by stratified squamous epithelium. Laminated keratin flakes were seen within the lumen.

depending on the availability of potential space in the area of the cyst. Giant epidermoid cysts have been reported in the scalp ${ }^{1}$, as well as in less hairy sites of the body such as the

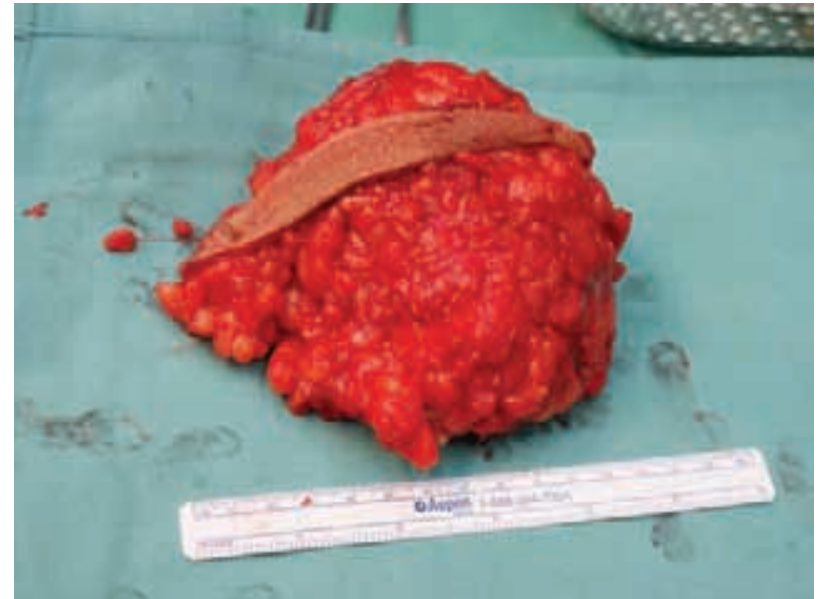

Fig. 2: Mass excised from posterolateral aspect of the right thigh.

buttoc $^{2,3}$ and forearm ${ }^{4}$. In our patient, presentation was in the lateral aspect of the right thigh, a relatively hairless area.

Diagnosis of sebaceous cyst is purely clinical and requires no imaging. In our patient, due to its enormous size, the lump mimicked a soft tissue sarcoma. MRI was required to verify any malignant features prior to a decision for tissue biopsy or surgical excision.

Epidermoid cyst is a benign condition requiring no treatment if it is asymptomatic. Infection of the cyst wall and the surrounding tissue may produce a boggy painful discharging lump known as Cock's peculiar tumour. An infected cyst requires a course of antibiotic followed by excision. However, there have been reports of malignant transformation of the lining of the cysts ${ }^{3,5}$.

Arianayagam et al..$^{5}$ reported a case of malignant epidermoid cyst in a 59-year-old female with inguinal node metastasis. Histopathology examination revealed an area of well differentiated squamous cell carcinoma within the cyst wall. The patient died six months later. 


\section{REFERENCES}

1. O’Brien J, Buckley O, Benfayed W, Torreggiani WC. Images in clinical radiology. JBR-BTR. 2008; 91: 64.

2. Polychronidis A, Perente S, Botaitis S, et al. Giant multilocular epidermoid cyst, on the left buttock. Dermatol Surg. 2005; 31(10): 1323-4.

3. Debaize S, Gebhart M, Fourrez T. et al. Squamous cell carcinoma arising in a giant epidermal cyst: a case report. Acta Chir Bel.g 2002; 102(3): 196-8.

4. Karadeli E, Kayahan Ulu EM, Ozgur AF, Tosun E. Giant Epidermal cyst of the forearm. Marmara Med J. 2009; $22(3)$ : $237-9$.

5. Arianayagam S, Javalakshmi. Malignant epidermal cyst: A case report. Malaysia J Pathol 1987; 9: 89-91. 